\title{
ADAPTACIÓN DE LAS PÁGINAS WEB DE LOS HOTELES EN LOS DISPOSITIVOS MÓVILES
}

\section{ADAPTATION OF WEB PAGES OF HOTELS IN MOBILE DEVICES}

Sánchez Jiménez, Miguel Ángel (Universidad de Cádiz) *

Correia, Marisol B. (Universidad de Algarve) ${ }^{* *}$

de Matos, Nelson (Universidad de Algarve) **

\section{Resumen}

En la actualidad, resulta esencial el concepto de web responsive que permite adaptar el contenido de las páginas web al dispositivo móvil mejorando así la experiencia del consumidor móvil. En este contexto se considera de especial relevancia la oferta turística siendo uno de los sectores en el que los usuarios buscan más información y realizan más compras a través de los dispositivos móviles. En este estudio se ha analizado la adaptación de las páginas webs de los hoteles de la provincia de Cádiz a los dispositivos móviles, para ello se han identificado las principales variables para conseguir un web responsive: la adaptación del tamaño de la web, la accesibilidad, la usabilidad y la posibilidad de comprar/reservar en la pantalla de inicio y se ha llevado a cabo una valoración subjetiva de las mismos. Los resultados en general son positivos, no obstante, se ha demostrado que aún las páginas webs de los hoteles no se han adaptado completamente a estos dispositivos, destacando la escasa visibilidad y tamaño del texto, así como la baja disponibilidad del apartado de reserva en la página de inicio.

Palabras claves: dispositivos móviles, web responsive, sector hotelero, páginas webs, experiencia.

\begin{abstract}
Mobile devices have become an essential element for users. This fact cannot go unnoticed by brands and business participants, who see a great opportunity to address consumers, thus fulfilling one of the maxims of marketing, in which these participants must be where the consumers are. In this way, the concept of responsive web is essential, which allows the content of the web pages to be adapted to the mobile device, thus improving the mobile consumer experience. In this context, the touristic offer is considered of special relevance being one of the sectors in which users seek more information and make more purchases through mobile devices. Thus, in this study we have analyzed the adaptation of the websites of the hotels in the province of Cádiz to mobile devices. The results in general are positive, however, it has been shown that the web pages of the hotels have not been fully adapted to these devices yet, highlighting the low visibility and size of the text, as well as the scarce availability of the reservation section in the homepage.
\end{abstract}

* Departamento de Marketing y Comunicación, Facultad de Ciencias Sociales y Comunicación, Campus Universitario de Jerez, 11405. miguelangel.sanchez@uca.es.

** Escola Superior de Gestão, Hotelaria e Turismo, Universidade do Algarve \& CiTUR Algarve, Centro de Investigação, Desenvolvimento e Inovação em Turismo \& CEG-IST, Instituto Superior Técnico, Universidade de Lisboa. mcorreia@ualg.pt.

*** Cieo, Escola Superior de Gestão, Hotelaria e Turismo, Universidade do Algarve, nmmatos@ualg.pt. Recibido: 21 de noviembre de 2017. Aceptado: 23 de abril de 2018. 
Keywords: mobile devices, responsive web, hotel sector, websites, experience.

\section{INTRODUCCIÓN}

La creciente importancia de las nuevas tecnologías y el marketing digital hace necesario el estudio del impacto que tendrán estos aspectos en el negocio empresarial, más concretamente en aquellos sectores que son más importantes en nuestro país. El Foro Económico Mundial (2017) especifica que el turismo es el sector más importante en nuestro país y que España cuenta con la economía mejor preparada para el turismo obteniendo el mayor índice de competitividad de viajes y turismo. Además, se especifica que la clave para conseguir el primer puesto este año ha sido el desarrollo digital que ha vivido el sector turístico español, destacando "la habilidad para apoyar digitalmente la búsqueda de entretenimiento”, lo cual refleja cómo el país se ha adaptado a los hábitos de consumo llegados con la revolución digital. Dentro del futuro del sector turístico señala la importancia en el desarrollo de las tecnologías de información y comunicación (TIC) como uno de los retos pendientes. Por último, señala la necesidad de que el sector se adapte rápidamente al marketing digital y cómo internet en el móvil define cada vez más cómo los viajeros seleccionan, planifican y evalúan sus viajes. Por lo tanto, queda claro que resulta esencial señalar la necesidad de que los países se adapten a las nuevas tendencias del turismo.

Dentro de estas nuevas tecnologías de la comunicación nos vamos a centrar en aquella que se da a través de los nuevos dispositivos móviles inteligentes, en el que el marketing móvil y el uso de aplicaciones móviles han cobrado una gran importancia en estos últimos años. IAB Spain (2017) resalta esta importancia del marketing móvil, en el que la inversión en este ámbito ha crecido una media del 65 por 100 anualmente. Además, resalta otros puntos que hacen denotar la necesidad de tener en cuenta el concepto móvil: La alta penetración de estos dispositivos (alcanzando actualmente el 87 por 100 de personas con un smartphone), el gran aumento del tiempo medio de conexión a través de estos dispositivos, el mayor uso de estos dispositivos en detrimento de otros más tradicionales como el ordenador de sobremesa o portátiles, el uso del móvil en el proceso de decisión de compra, el aumento de la presencia de las marcas y, el aspecto que más nos interesa en este estudio, la búsqueda a través de los dispositivos móviles del ocio y viajes, lo cual vuelve a destacar la importancia que tiene esta nueva tecnología en el sector turístico.

En este contexto, resulta esencial estudiar cómo las páginas web de productos turísticos se han adaptado a estos nuevos dispositivos móviles. El concepto denominado web responsive consiste en aplicar un diseño a la página web que le permite adaptarse a todo tipo de resoluciones, ya sea la de un móvil, una tableta, pantallas widescreen o sea cual sea la resolución requerida (Aubry, 2014). En este sentido resulta esencial que el contenido sea accesible y que los productos y servicios electrónicos puedan ser utilizados por los usuarios con efectividad, eficiencia y satisfacción en un contexto de uso determinado (Mascaraque et al., 2009). La web responsive ayuda a que este acceso a las páginas web provoque una mejor experiencia en el usuario, adaptando su contenido en todos los dispositivos. De esta manera la web responsive mejoraría la visibilidad en todos los dispositivos para páginas web sobre destinos turísticos, agencias de viajes u ofertas turísticas como hoteles, restaurantes, lugares de interés, etc. Destacando además que muchos de estos servicios son contratados on line a través de la página web, por lo tanto, la experiencia del usuario para poder contratar o reservar dichos servicios turísticos en cualquier dispositivo debe ser la idónea. 
En los últimos años, el sector hotelero, dado el alto peso que supone sobre el total de las empresas turísticas, se ha convertido en una de las ramas del ámbito terciario de mayor relevancia e importancia de su economía desempeñando un papel fundamental en la salida de la crisis económica del país (Miranda et al., 2016). Por lo tanto, aunque esta tecnología se ha aplicado en webs de todos los sectores, su utilización se acentúa en el sector hotelero debido a la portabilidad y accesibilidad de la información, aspectos claves para los turistas que desean informarse y contratar los servicios de un hotel a través de los dispositivos móviles.

El sector turístico, en general, y el sector hotelero en particular, tienen una gran importancia en la provincia de Cádiz, siendo de los principales dinamizadores de la economía y empleo de la provincia, ostentando un papel esencial en el PIB provincial (Diputación de Cádiz, 2017). Ante el gran aumento de la utilización de los dispositivos móviles se ha convertido en una prioridad para las empresas turísticas aprovechar esta oportunidad para mantener la competitividad y superar las posibles amenazas, circunstancia que debe ser tenida en cuenta por el sector hotelero de la provincia de Cádiz.

De esta manera, en este estudio se va a comprobar cómo se han adaptado las páginas web de los hoteles de la provincia de Cádiz de 3, 4 y 5 estrellas a los dispositivos móviles. Para ello, se han identificado las principales variables referidas a la buena utilización del web responsive como son el tamaño del contenido, la accesibilidad o visibilidad del contenido y a la usabilidad. De esta manera se pretende comprobar si las páginas web de los hoteles de la provincia de Cádiz se han adaptado eficientemente a los dispositivos móviles y cuáles son los principales problemas que se han detectado al respecto.

A continuación, se va a especificar el marco teórico del estudio, primero se determina la trascendencia del comercio electrónico en la oferta turística, posteriormente se especifican las particularidades del sector hotelero como objeto de estudio para, finalmente, conocer las principales especificaciones sobre el web responsive. Tras el marco teórico se establece la metodología a seguir para alcanzar el objetivo y, posteriormente, se establecen los principales resultados. Para finalizar, en el último apartado se establecen las principales conclusiones del estudio.

\section{MARCO TEÓRICO}

\subsection{El comercio electrónico en la oferta turística}

Desde que surgió internet, las empresas turísticas lo adoptaron rápidamente debido a las ventajas potenciales que este nuevo sistema tenía, sobre todo como medio de comunicación (López et al., 2009).

Entre las ventajas que ha tenido la integración de internet con el turismo cabe citar las siguientes (González, 2011):

a) Accesibilidad: los turistas pueden navegar desde casi cualquier parte del planeta, durante las veinticuatro horas del día.

b) Comodidad: no resulta incómodo disponer de internet ni realizar las conexiones y la navegación. Las empresas hoteleras han ofrecido la conexión a internet como un producto más, o como un servicio diferenciador.

c) Rapidez: internet es cada vez más veloz. La obtención y el envío de cualquier tipo de dato se realiza en un corto periodo de tiempo. Esto ha agilizado la confección, comercialización y disfrute de infinidad de productos turísticos. 
d) Novedad: internet se puso rápidamente de moda, y lo sigue estando. Esto ha facilitado su rápida extensión. De hecho, cuando internet se ha incorporado a las experiencias de los diferentes sectores éstos se han puesto de moda.

e) Cobertura global: la conexión a través de internet se puede establecer con cualquier parte del mundo, lo cual constituye una ventaja que está en consonancia con las características y dinámicas del propio sector turístico.

f) Gran riqueza: internet es multimedia, pues integra imagen, sonido e interacción. Este hecho construye una ventaja de gran valor para promocionar destinos y productos turísticos.

g) Flexibilidad: permite actuaciones al mismo tiempo masivas y personalizadas. Por otra parte, se pueden realizar ajustes y actualizaciones en cualquier momento.

h) Bajo coste: integrar internet en la empresa no requiere grandes inversiones, ni de tiempo ni de recursos monetarios.

Las TIC e internet han cambiado la manera en que se informa sobre el turismo (Buhalis y Law, 2008) y la forma en que las compañías y comunidades turísticas trabajan en el diseño y la comercialización de sus productos, del mismo modo en que se ha transformado la forma en que los turistas planifican, reservan y viven sus experiencias turísticas (Munar, 2012). No hay duda que el escenario ofrecido por internet y las TIC ha favorecido la incorporación de diversos recursos y procesos que generan información sobre las preferencias en el consumo del turista, ofreciendo una respuesta a las necesidades relacionadas con la información y, por lo tanto, mejorando la competitividad de las ofertas turísticas de los destinos (Leung y Tanford, 2016).

Ferrá y Cardona (2015) consideran que las TIC forman parte de la cultura actual y amplían las posibilidades de desarrollo social personal. Suelen incluirse en el concepto TIC no solamente la informática y sus tecnologías asociadas, telemática y multimedia, sino también los medios de comunicación de todo tipo: los medios de comunicación social y los medios de comunicación interpersonales tradicionales con soporte tecnológico como el teléfono. Las TIC contribuyen a la rápida obsolescencia de los conocimientos y a la emergencia de nuevos valores, provocando continuas transformaciones en las estructuras económicas, sociales y culturales, e incidiendo en casi todos los aspectos de la vida. Su gran impacto en todos los ámbitos hace cada vez más difícil que se pueda actuar eficientemente prescindiendo de las TIC.

De esta forma surge el termino de turismo electrónico, que incluye el diseño, la implementación y la aplicación de las TIC y soluciones de comercio electrónico en la industria del turismo, el análisis del impacto de los procesos económicos y técnicos, así como las estructuras de mercado de todos los actores involucrados en las experiencias de los viajeros (Werthner et al., 2015).

En el estudio de Moreno et al. (2016) se considera que el turismo electrónico reúne a todas las TIC que se aplican a la industria del turismo, y también refleja la digitalización de los procesos y las cadenas de valor en los viajes de turismo, hostelería y catering. El turismo electrónico incluye todas las funciones de la empresa tales como comercio, marketing, relación con el cliente, finanzas, gestión de los recursos humanos, contabilidad, estrategia, planificación y gestión electrónica para todos los sectores de la industria del turismo, es decir; viajes, transporte, ocio, hospitalidad. La información es la esencia del turismo, por esta razón, la tecnología es fundamental para que la industria funcione. Hoy en día, las TIC son una necesidad para la comercialización, distribución, 
promoción y coordinación de la industria del turismo. Si se utilizan de manera eficaz, el internet y las TIC permiten a las compañías de turismo (Moreno et al., 2016):

- Interactuar eficientemente con sus clientes y personalizar sus productos y servicios.

- Desarrollar el turismo de intermediación y aumentar los puntos de venta, por ejemplo, el sitio web de la propia empresa, Booking, Expedia, etc. (principalmente para empresas de hostelería).

- Permitir a los clientes comunicarse con otros usuarios, por ejemplo, a través de Tripadvisor en el perfil de la empresa.

- Proporcionar servicios basados en la ubicación, integrando datos, contenidos e información multimedia en Google Maps.

Respecto al sector hotelero, es importante saber cómo funcionan las TIC. Causado et al. (2015) especifican que las TIC son utilizadas en el sector hotelero para ampliar sus operaciones, gestionar su inventario y maximizar su rentabilidad. Además, los hoteles también utilizan las TIC y el internet para vender sus productos o servicios y comercializar su empresa. La presencia online es necesaria para permitir, tanto a clientes particulares como a las grandes empresas de comercio de viajes, acceder a información precisa sobre la disponibilidad y proporcionar un servicio sencillo, eficaz, barato y fiable sobre los procesos relacionados con el mismo y confirmando las reservas (Cooper, 2008).

\subsection{El sector hotelero}

Una de las principales actividades del turismo en España es la hotelera, la cual está inmersa en profundas transformaciones derivadas de los cambios sociales y tecnológicos que afectan a la gestión y a la prestación del servicio y a la forma de comercializar el producto hotelero (Rodríguez et al., 2010). A continuación, se van a analizar las características principales y la situación actual del sector hotelero en España.

En los últimos años, el sector hotelero, dado el alto peso que supone sobre el total de las empresas turísticas, se ha convertido en una de las ramas del ámbito terciario de mayor relevancia e importancia de la economía española, desempeñando un papel fundamental en la salida de la crisis económica (Miranda et al., 2016).

El Real Decreto 1.634/83 de 15 de junio, capitulo II, artículo 6, por el que se regula las normas de clasificación de los establecimientos hoteleros, define los hoteles como "aquellos establecimientos que, ofreciendo alojamiento, con o sin comedor, y otros servicios complementarios, ocupan la totalidad de un edificio o parte independizada del mismo, constituyendo sus dependencias un todo homogéneo, con escaleras y ascensores de uso exclusivo y que reúnen los requisitos técnicos mínimos que establece la ley".

El sector hotelero posee algunas características particulares que deben considerarse, tales como (Rojo, 2014):

- Intangibilidad del producto principal que genera, el cual no se puede almacenar. El producto hotelero no es intangible, pero si perecedero, y además adquiere un papel fundamental la manera intangible de ofrecer el servicio. 
- El factor localización adquiere más importancia, al ser más determinante que en otro tipo de empresas.

Como en cualquier otro tipo de empresa, en los hoteles se llevarán a cabo funciones de aprovisionamiento, producción, comercialización, financiación, gestión del personal y administración; pero, además, cabe mencionar las siguientes actividades específicas que con carácter general se realizan en estos establecimientos, y que componen su producto/servicio (Rojo, 2014):

- Servicio de recepción; Se encarga de las reservas de plazas, atender la entrada y salida de huéspedes y clientes, mantener relaciones públicas con clientes, coordinación interdepartamental, realización y cobro de facturas, etc.

- Servicio de conserjería: se ocupa de ofrecer información y asistencia a los huéspedes y clientes, con su equipaje, consignas, custodia de llaves de las habitaciones, correspondencia, etc.

- Pisos: realiza la limpieza y preparación de las habitaciones, conservación del mobiliario, limpieza de pasillos salones y zonas comunes, control de stock e inventario de tipo de habitaciones, lavandería y plancha, etc.

- Economato y Bodega: lleva a cabo las compras de productos de economato y bodega, almacenamiento de esta mercancía, distribución a los distintos departamentos.

- Cocina y Restauración: entre las principales funciones destacan el servicio, la manipulación de los alimentos, etc.

- Administración y Contabilidad: lleva a cabo la fijación de los objetos generales de la compañía, el control de todos los ingresos y gastos de la explotación, la evaluación y selección de inversiones, la elección de las distintas fuentes de financiación de la compañía.

- Comercialización: se encarga de la realización de estudios de mercado, campañas de publicidad, relaciones con empresas de intermediación turística, etc.

En cuanto a la clasificación de su oferta o tipologías de empresas hoteleras, se pueden citar las siguientes como las más significativas (García, 2014):

- Según el establecimiento hotelero: hoteles, hostales y pensiones.

- De acuerdo con su localización: de ciudad, de montaña, de costa, de carretera, etc.

- En función de su categoría: una, dos, tres, cuatro y cinco estrellas.

- Según la dimensión: de gran tamaño (más de 300 habitaciones), de tamaño intermedio (de 100 a 300 habitaciones) y de pequeño tamaño (menos de 100 habitaciones).

- Por la forma de operación y su relación con la propiedad: explotadas directamente por la propiedad, explotadas de forma mixta y explotadas en régimen de franquicia.

- Según la forma de producción: producción continua o establecimientos que permanecen abiertos ininterrumpidamente y producción discontinua 0 establecimientos de temporada. 


\subsection{Web responsive}

La web responsive consiste en aplicar un diseño a la página web que le permite adaptarse a todo tipo de resoluciones, ya sea la de un móvil, una tableta, pantallas widescreen, sea cual sea la resolución requerida.

Según el estudio de González y Marcos (2013), existen páginas web que utilizan múltiples canales para llegar a la diversa gama de dispositivos disponibles en el mercado. Las aplicaciones móviles son sólo algunas de las alternativas que se han venido llevando a cabo a lo largo de estos años. Sin embargo, existe una dificultad a la hora de desarrollar e implementar este tipo de canales al realizar un esfuerzo a nivel técnico, ya que en muchos casos se parte desde cero.

Esta situación parece estar cambiando. La tendencia actual es reducir la cantidad de canales que tienen las diferentes páginas web, aprovechando un único canal cuya estructura flexible le permita adaptarse a ordenadores, televisores, tabletas o teléfonos móviles, con sus respectivos tamaños de pantalla. Es lo que se conoce como web responsive.

En este momento nos encontramos con cada vez más tipos de dispositivos, modos de entrada, y navegadores que nunca. De ahí la importancia de tener una navegación multiplataforma óptima, con la web responsive como referencia del diseño web en la actualidad.

En este mismo artículo se relaciona la web responsive con la experiencia al usuario. La norma ISO 9241-210 la define como "la percepción de una persona y las respuestas que resultan del uso previsto de un producto, sistema o servicio". La experiencia del usuario ha evolucionado junto con las nuevas tecnologías y los diferentes tipos de usuarios. Inicialmente solo contaba con un dispositivo de interacción específico, los ordenadores, hoy día vemos multitud de nuevos dispositivos que permiten al usuario estar conectado en cualquier lugar y momento, un principio de ubiquidad que poco a poco va cambiando la forma de pensar la web. Por lo tanto, la web responsive se considera una parte esencial en el entorno digital hoy en día para generar una mejor experiencia de usuario es cuestión de adaptarse a los nuevos patrones de consumo y responder a las necesidades de los usuarios, ofreciendo sitios web que proporcionen una experiencia de usuario igual de satisfactoria indistintamente del dispositivo desde el cual naveguen.

Furroy (2015) considera que el web responsive o diseño adaptativo consiste en "diseñar sitios web de tal manera que el mismo pueda adaptarse a los diferentes dispositivos que utilizan los usuarios a la hora de navegar por internet, ya sean tabletas, smartphones, ordenadores, etc." En internet la clase no es sólo el diseño de una web sino conseguir la correcta adaptación visual y de contenidos en todos los dispositivos por igual.

En el estudio de Baturay y Birtane (2013) se tratan también diversas consideraciones sobre web responsive. En él se comenta que cada vez que un usuario entra en una página web, lo más importante y fundamental que él busca es si puede acceder a toda la información que necesita tan pronto como le sea posible con el mínimo esfuerzo. Esto requiere el suministro de la mejor experiencia para el usuario con un mínimo cambio de tamaño y desplazamiento durante la navegación de la página web (Sharkie y Fisher, 2013). Hay una necesidad de cambiar a un nuevo diseño web que es capaz de remodelar en función de diversos tamaños de pantalla y resoluciones desde mayores tamaños de pantalla hasta otras más pequeñas en los dispositivos 
móviles. Por lo tanto, los usuarios estarían expuestos a una mejor experiencia de visualización de contenido en la pantalla del dispositivo o plataforma que estén viendo.

El término web responsive fue acuñado en 2010 en la página web “A book apart”. Aquí considera que la web responsive comprende una serie de técnicas y pautas de diseño que permiten adaptar páginas web al entorno de navegación del usuario, entendiendo como entorno de navegación la multiplicidad de dispositivos, móviles o no, por medio de los cuales los usuarios pueden acceder y navegar en internet.

Desde entonces, muchos proyectos se han desarrollado utilizando sus técnicas (Rekhi, 2013). El término se utiliza a menudo para referirse a un significado que tiene otras denominaciones como: fluid design, elastic layout, rubber layout, liquid design, adaptative layout, cross-device design y flexible design.

El web responsive permite a los usuarios la mejor experiencia mientras navegan por las páginas web a través de un mundo de múltiples dispositivos tales como teléfonos inteligentes, tabletas, ordenadores portátiles y ordenadores de escritorio. Es hora de que los diseños de las páginas web deban permitir que el usuario responda adecuadamente sea cuál sea el dispositivo que esté utilizando (Sharkie y Fisher, 2013). Rekhi (2013) afirma que el trabajo de los diseñadores no es muy importante en la fase de desarrollo de un sitio web adaptable que utiliza diferentes resoluciones de pantalla, ya que con el lenguaje de programación utilizado (CSS3) y sus técnicas de diseño resulta cada vez más sencillo.

En el trabajo de Furroy (2015) se recoge la importancia de la web responsive en el sector hotelero. Comenta que gran parte de los viajeros de todo el mundo utilizan dispositivos móviles para navegar en webs de hoteles o realizar reservas hoteleras, cifra que crece exponencialmente año tras año. De esta manera, disponer de una web responsive es una prioridad de cualquier empresario del sector turístico.

Además, va un paso más allá, considera que la adaptación web al móvil a través del web responsive no es suficiente, ya que sería lo básico. Las nuevas generaciones de usuarios utilizan y realizan cada vez más tareas a través de los dispositivos móviles, cada vez más utilizan sus smartphones para consultar, comparar ofertas, ver imágenes y adquirir distintos productos. El sector turístico y hotelero no puede mostrarse ajeno a esta tendencia. Por lo tanto, buscan en estos dispositivos una experiencia de reserva completamente adaptada a las características y comportamientos asociados con los smartphones y tabletas. El sector hotelero debe de dar un paso más y contar con un motor de reservas completamente móvil que ayude a simplificar los pasos al mismo tiempo que funciona como herramienta de marketing.

Una muestra de esto es que las principales páginas web o actores del sector (como TripAdvisor, Booking, Expedia o Kayak) se han adaptado a este entorno móvil rediseñando sus aplicaciones y lanzando otras nuevas con productos y herramientas pensadas específicamente para el último minuto.

\section{METODOLOGÍA}

En este estudio se ha llevado a cabo un análisis para comprobar si las páginas webs de los hoteles de la provincia de Cádiz están adaptadas a los dispositivos móviles. Para ello se han identificado las principales variables o factores para conseguir un buen web responsive y que contribuyen a mejorar la experiencia del usuario: la adaptación del tamaño de la web, la accesibilidad, la usabilidad y la posibilidad de comprar/reservar en la pantalla de inicio. Así, la aparición de dispositivos móviles con mayores capacidades y funciones dieron lugar a nuevas tendencias del diseño web 
buscando adaptar las páginas a diferentes tamaños de pantalla y mejorar la usabilidad y la accesibilidad para el usuario (Parra Luzuriaga, 2015).

El cambio constante de tamaño de pantalla en dispositivos móviles ha generado que los sitios web busquen la forma más apropiada para adaptar la información a mostrar, por lo que se considera esencial para conseguir un buen web responsive la correcta adaptación del contenido al tamaño de la pantalla en estos dispositivos (Arce, 2016). Así, al visualizar la web a través de una pantalla pequeña de móvil se mantendrán los elementos principales de la misma, rediseñados, reorganizados, proporcionales entre ellos y con respecto al tamaño original (González y Marcos, 2013). En este sentido, para conseguir una correcta adaptación es clave la no necesidad de realizar scroll horizontal ni zoom. El desplazamiento causa problemas, sobre todo por el uso excesivo. El problema se agrava cuando el desplazamiento es en varias direcciones por el tamaño de la pantalla que hace que el usuario pierda la noción de dónde se encuentra. Así, la página debe estar diseñada de una manera simple, que el desplazamiento en una sola dirección (generalmente vertical) permita al usuario experimentar todo su contenido. Por lo tanto, es mejor realizar más pasos que usar el scroll horizontal. Se evita el envío de información a cambio de la realización de más peticiones, permitiendo de este modo que el usuario interactúe mejor con la información y obtenga resultados más precisos y sintetizados. (Parra Luzuriaga, 2015; Rodríguez et al., 2017).

La accesibilidad es otro de los principales factores a tener en cuenta. La Accesibilidad es el grado en el que todas las personas pueden utilizar un objeto, visitar un lugar o acceder a un servicio, independientemente de sus capacidades técnicas, cognitivas o físicas (INTECO, 2009). Martín et al. (2012) señala que accesibilidad web está dirigida a lograr una web de todos y para todos, independientemente de las discapacidades permanentes o temporales, los problemas asociados a la edad, las brechas generacionales, las habilidades y preferencias de las personas, la cultura y el nivel educacional alcanzado, etc. También destaca que una mala experiencia del usuario en el entorno web puede tener efectos bastantes negativos y difíciles de revertir. En el ámbito de estudio, la accesibilidad de dispositivos móviles sigue los mismos principios esenciales que la accesibilidad web, pero teniendo en cuenta las particularidades que ya hemos comentado acerca de los dispositivos móviles. Según González (2013) esta definición se refiere a que los elementos que componen la interfaz del usuario de la aplicación deben ofrecer una determinada información para que los servicios de accesibilidad que funcionan en el sistema operativo o los productos de apoyo (software o hardware), puedan interactuar correctamente y permitan el acceso del usuario al dispositivo. Completando la definición puede decirse que una aplicación es accesible cuando cualquier usuario, independientemente de su diversidad funcional, puede utilizarla en su dispositivo móvil satisfactoriamente con su sistema de acceso habitual. El World Wide Web Consortium (W3C) ha desarrollado a través de la Web Accessibility Initiative (WAI), recomendaciones sobre la accesibilidad web y móvil, entre ellas destaca la necesidad de una adecuada visibilidad y tamaño de los elementos del contenido ya sean imágenes, textos, enlaces, botones, etc.

En lo que respecta a la usabilidad es considerada uno de los factores más importantes dentro de la calidad de un producto de software (Enriquez y Casas, 2014). La usabilidad se considera como un atributo de calidad de una página web, que determina la facilidad de la interfaz para ser utilizada. Así, se evalúan los aspectos referentes a la facilidad de uso de la página, adaptado a las demandas de los usuarios. Si se incluyen aspectos de usabilidad, el usuario final del sistema podría interactuar con el 
mismo logrando realizar tareas de una manera fácil y eficiente (Nielsen y Budiu, 2013). Según Nielsen y Loranger (2006) la usabilidad puede definirse como la facilidad de uso, más específicamente, hace referencia a la rapidez con que se puede aprender a utilizar algo, la eficiencia al utilizarlo, cuál es su grado de propensión al error, y cuánto les gusta a los usuarios. Sánchez (2012) considera la usabilidad en las aplicaciones móviles la navegación por la aplicación, analizada conforme a la presencia de menú de secciones, navegación dentro de la misma sección, claridad en la estructura de navegación, etc., y es un aspecto esencial para la calidad de la aplicación.

Por último, destacar la importancia de la página de inicio en las páginas web de los dispositivos móviles. El objetivo de esta página de inicio es conseguir que el visitante de la web genere una acción, completando el evento de conversión. Para un hotel, en la mayoría de los casos, la pagina de inicio es una de venta directa y es creada para que los usuarios realicen una reserva. la finalidad de esta página de inicio es que el usuario pulse el botón de "suscríbete”, “compra” o "reserva”. Por ello hay que insertar un par de botones, destacándolos y haciéndolos comprensibles con pocas palabras (Hostalia, 2012; Hostelsur, 2017).

Para comprobar si se cumplen eficientemente estas variables se utiliza una valoración subjetiva de la utilización del recurso (Shneiderman, 2010; Varela y Shear, 2005), en este caso se ha comprobado si se implementan las variables accediendo y examinando las páginas webs de los hoteles a través de los dispositivos móviles. Para ello, se ha tomado como referencia una serie de ítems.

En el cuadro 1 se especifican las variables e ítem que se utilizan en el estudio.

\begin{tabular}{|c|l|l|}
\hline \multicolumn{2}{|c|}{ CUADRO 1. CRITERIOS UTILIZADOS PARA EL ANÁLISIS } \\
\hline Variables & \multicolumn{1}{|c|}{ Descripción } & \multicolumn{1}{c|}{ Referencia } \\
\hline $\begin{array}{c}\text { Adaptación del } \\
\text { tamaño de la web }\end{array}$ & $\begin{array}{l}\text { Tamaño del contenido de la pantalla } \\
\text { adaptado al dispositivo móvil sin } \\
\text { necesidad de realizar scroll horizontal } \\
\text { ni zoom }\end{array}$ & $\begin{array}{l}\text { González y Marcos } \\
\text { (2013); Parra Luzuriaga } \\
\text { (2015); Rodríguez et al. } \\
\text { (2017) }\end{array}$ \\
\hline Accesibilidad & $\begin{array}{l}\text { Visibilidad del contenido (botones, } \\
\text { texto o imágenes) y el tamaño de los } \\
\text { elementos del contenido (texto, } \\
\text { imágenes y enlace de reserva) }\end{array}$ & $\begin{array}{l}\text { Zambrano et al. (2017); } \\
\text { W3C (2017) }\end{array}$ \\
\hline Usabilidad & $\begin{array}{l}\text { Navegación fácil e intuitiva a través } \\
\text { de la página web en el dispositivo } \\
\text { móvil }\end{array}$ & $\begin{array}{l}\text { Enriquez y Casas } \\
\text { (2014); Sánchez (2012); } \\
\text { Zambrano et al. (2017) }\end{array}$ \\
\hline $\begin{array}{c}\text { Reserva en la } \\
\text { pantalla de inicio }\end{array}$ & $\begin{array}{l}\text { Posibilidad de realizar la reserva del } \\
\text { hotel en la página de inicio en el } \\
\text { dispositivo móvil }\end{array}$ & $\begin{array}{l}\text { Hostalia (2012); } \\
\text { Hostelsur (2017) }\end{array}$ \\
\hline
\end{tabular}

Fuente: elaboración propia. 
El análisis fue llevado a cabo en las páginas web oficiales de todos los hoteles de la provincia de Cádiz de 3, 4 y 5 estrellas, que hacen un total de 79. Se estableció una base de datos con las páginas web de los hoteles y posteriormente se añadieron los resultados obtenidos en función de cada variable. La recolección de los datos y el establecimiento de los resultados se realizó accediendo a las 79 páginas web oficiales de los hoteles a través de un dispositivo móvil y valorando si se cumplían o no las especificaciones de las variables comentadas anteriormente. La recolección y análisis tuvo lugar durante los meses de junio y julio de 2017.

\section{RESULTADOS}

A continuación, se especifican los resultados obtenidos en el análisis de las páginas web de los hoteles de la provincia de Cádiz a través de los dispositivos móviles.

Respecto a la adaptación del tamaño de la web al dispositivo móvil se comprueba que 66 de las 79 páginas web de los hoteles (83,5 por 100) están correctamente adaptadas al tamaño del dispositivo móvil, no siendo necesario el desplazamiento scroll hacia los lados ni la generación de zoom para que el contenido este correctamente adaptado (gráfico 1).

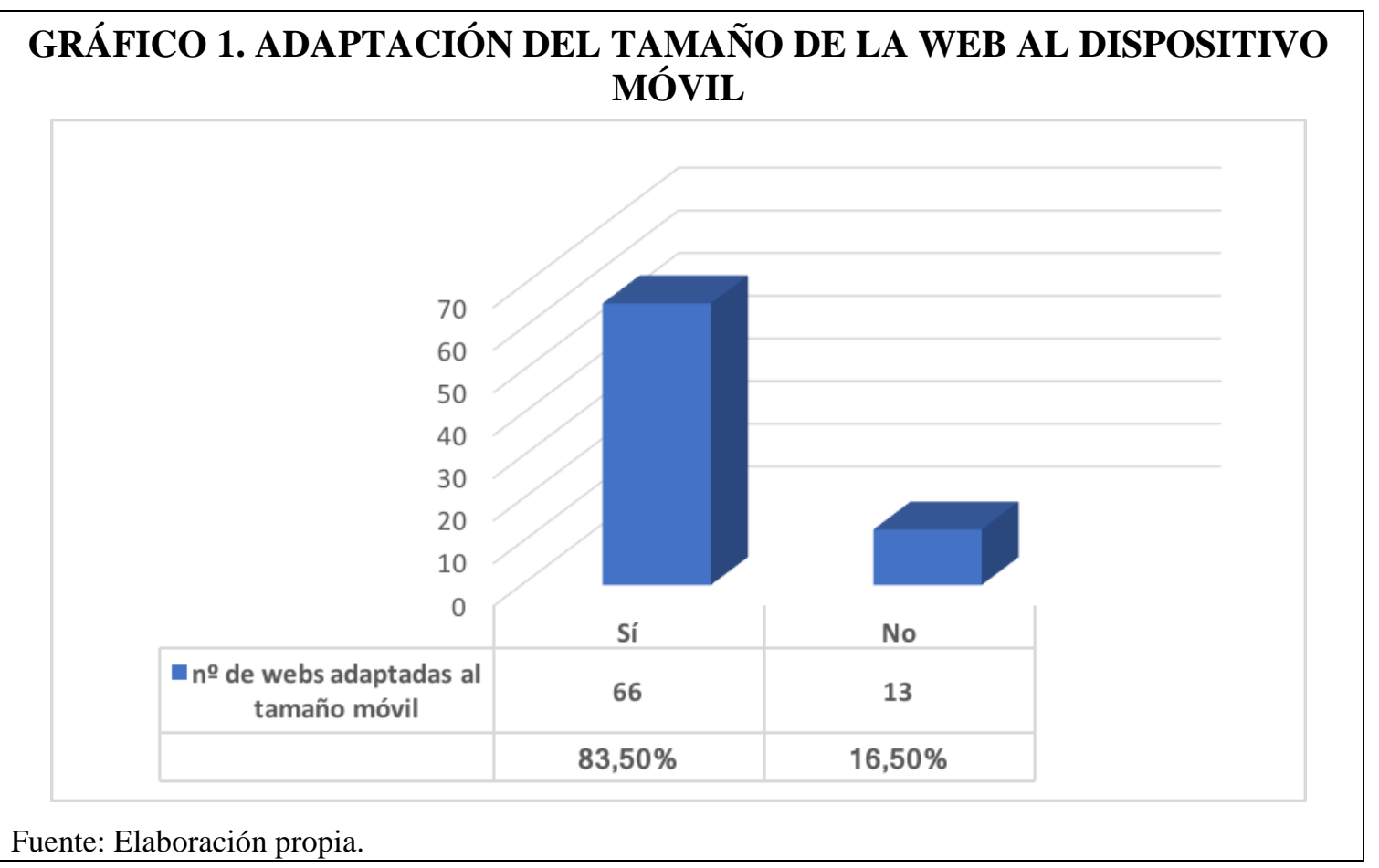

Para los resultados en la accesibilidad o visibilidad del contenido se tienen en cuenta las 66 páginas webs de los hoteles que están correctamente adaptadas en tamaño. En este sentido, se observa que 37 de los 66 hoteles tienen una escasa visibilidad del texto (56 por 100) (gráfico 2). También, cabe destacar que 15 páginas webs de los hoteles tienen una escasa visibilidad de los enlaces de reserva (22,7 por 100) (gráfico 3). 


\section{GRÁFICO 2. ESCASA VISIBILIDAD DEL TEXTO EN LOS DISPOSITIVOS MÓVILES}

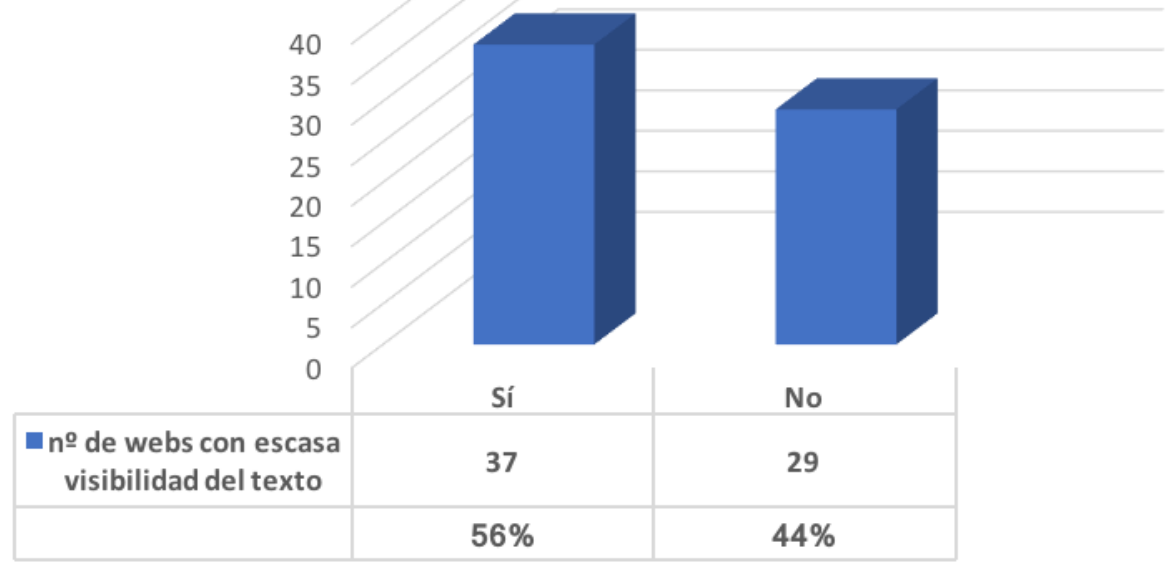

Fuente: Elaboración propia.

\section{GRÁFICO 3. ESCASA VISIBILIDAD DEL ENLACE DE RESERVA EN LOS DISPOSITIVOS MÓVILES}

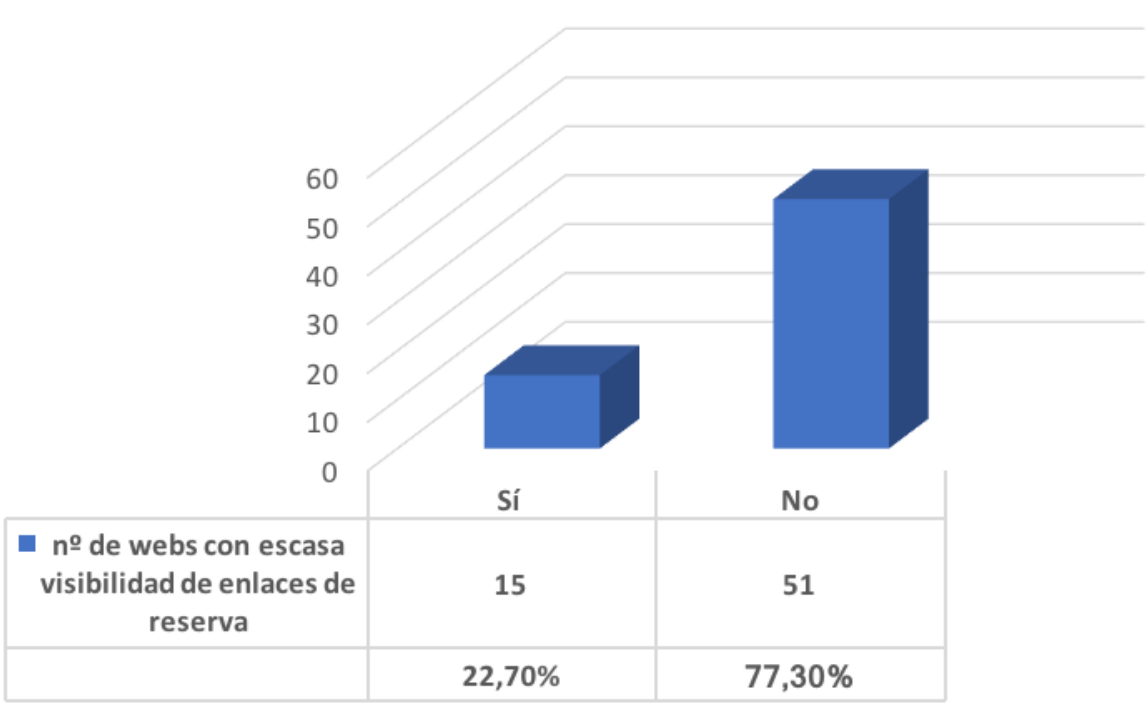

Fuente: Elaboración propia.

En lo que respecta a la usabilidad de la navegación en los dispositivos móviles de las páginas webs de los hoteles cabe destacar que la gran mayoría son usables siendo únicamente 15 páginas webs de las 66 las que se han considerado que disponen de un menú poco intuitivo (gráfico 4). 


\section{GRÁFICO 4. USABILIDAD DE LA NAVEGACIÓN EN LOS DISPOSITIVOS MÓVILES}

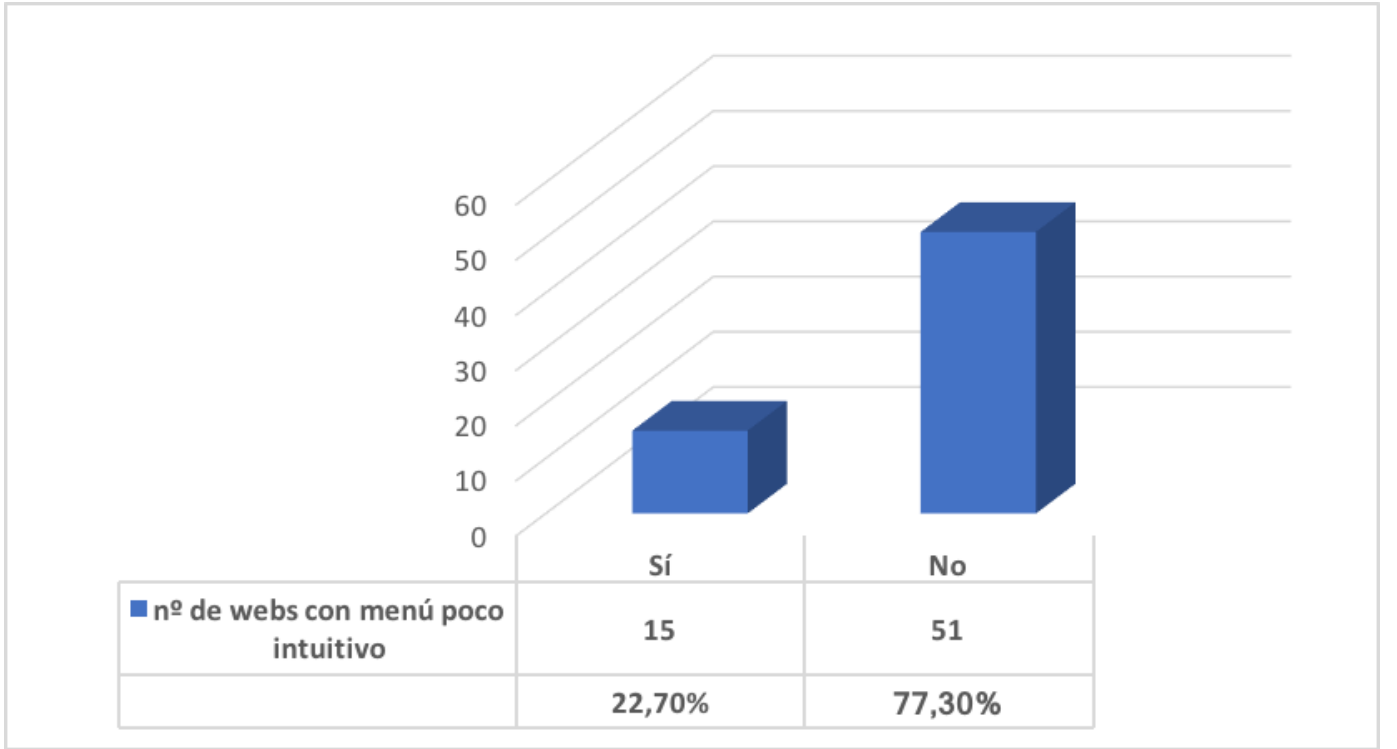

Fuente: Elaboración propia.

También se ha considerado de interés comprobar la posibilidad de reservar en la pantalla de inicio de las páginas webs de los hoteles en los dispositivos móviles, ya que mejora la experiencia del usuario y facilita la realización de una reserva a través de estos dispositivos. En este sentido se ha comprobado que solo 27 de las 66 páginas web (40,9 por 100) permiten la posibilidad de reservar en su página de inicio (gráfico 5).

\section{GRÁFICO 5. POSIBILIDAD DE RESERVAR EN LA PANTALLA DE INICIO EN EL DISPOSITIVO MÓVIL}

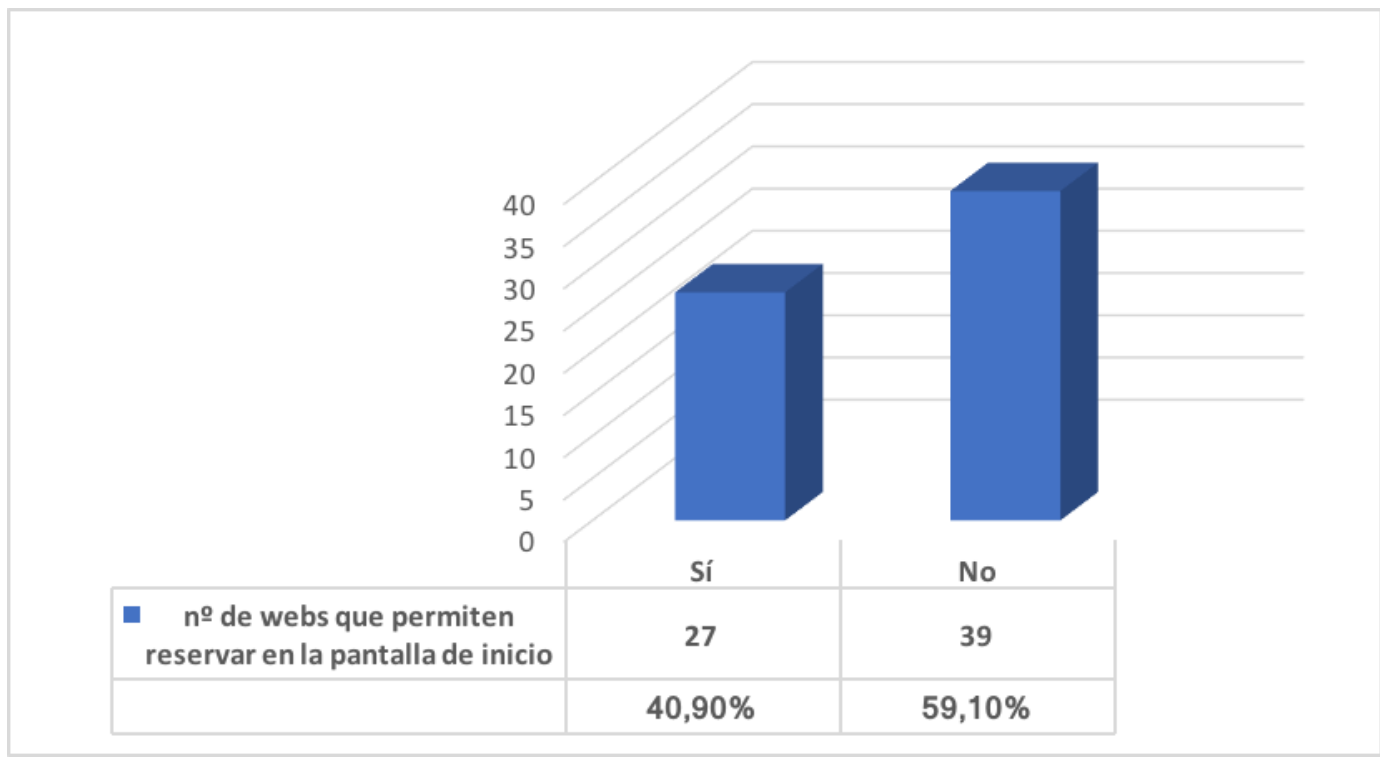

Fuente: Elaboración propia. 


\section{CONCLUSIONES}

Internet puede ser considerado como un gran mercado de usuarios a los que poder dirigirse. Además, muchas personas acuden a internet para informarse con todo lo relacionado al turismo. Por lo tanto, las páginas web sobre el sector turístico son de vital importancia para conseguir una buena promoción del destino o la oferta turística. La web responsive ayuda a que este acceso a las páginas web provoque una mejor experiencia en el usuario, adaptando su contenido en todos los dispositivos. De esta manera la web responsive mejoraría la visibilidad en todos los dispositivos para páginas web sobre destinos turísticos, agencias de viajes u ofertas turísticas como hoteles, restaurantes, lugares de interés, etc. Destacando además que muchos de estos servicios son contratados on line a través de la página web, por lo tanto, la experiencia del usuario para poder contratar o reservar dichos servicios turísticos en cualquier dispositivo debe ser la idónea.

En este estudio se ha podido comprobar que, aunque el 83,5 por 100 de los hoteles están adaptados al formato móvil, únicamente el 32,9 por 100 está totalmente optimizado y permite una experiencia idónea a través de dicho formato. Esto es especialmente importante ya que como se ha demostrado cada vez más consumidores utilizan estos dispositivos, tomando especial relevancia los productos turísticos, siendo uno de los sectores en el que los usuarios buscan más información y realizan más compras. Por lo tanto, aunque la mayoría de los hoteles de la provincia de Cádiz se ha preocupado por la adaptación de sus páginas webs, teniendo en cuenta la gran importancia de estos dispositivos, aún queda por maximizar esa adaptación resolviendo los problemas derivados de ella, maximizando así la experiencia del usuario.

El principal problema es la escasa visibilidad y tamaño del texto en dichos dispositivos, lo que obliga a realizar zoom en determinados momentos. También destacar la escasa disponibilidad de la posibilidad de realizar reserva en la página de inicio de la web, lo que facilitaría la obtención de información sobre los días de ocupación del hotel y la propia realización de la reserva.

A través de este estudio se ha podido comprobar cómo se adaptan las páginas web de los hoteles de 3, 4 y 5 estrellas de la provincia de Cádiz a los dispositivos móviles, así como la identificación de los principales problemas y factores de mejora al respecto. No obstante, como limitación del estudio cabe comentar que este estudio se ha realizado desde el punto de vista de las páginas web de las empresas hoteleras, sin contar con la opinión de los usuarios. De esta manera, como futura línea de investigación se propone realizar un análisis más exhaustivo del web responsive de las páginas web de los hoteles teniendo en cuenta la interacción y la opinión que realizan los propios usuarios.

\section{BIBLIOGRAFÍA}

Arce, A. E. V. (2016): “De la interfaz del usuario al responsive web design”, Revista $A U C, \mathrm{n}^{\circ}$ 37, pp. 59-66.

Aubry, C. (2014): HTML5 y CSS3: para sitios con diseño web responsive. Ediciones ENI, Barelona.

Baturay, M. H. y Birtane, M. (2013): "Responsive web design: A new type of design for web-based instructional content”, Procedia-Social and Behavioral Sciences, $\mathrm{n}^{\circ}$ 106, pp. 2275-2279. 
Buhalis, D. y Law, R. (2008): "Progress in information technology and tourism management: 20 years on and 10 years after the internet - the state of eTourism research”, Tourism Management, vol. 29, n 4, pp. 609-623.

Causado Rodríguez, E., García Guiliany, J., Martínez Ventura, J. y Herrera Flórez, A. (2015): Tecnologías de información y comunicación en el sector hotelero, Corporación Universitaria Latinoamericana, Barranquilla.

Cooper, C. (2008): Tourism: Principles and practice. Pearson education, Londres.

Diputación de Cádiz (2017): “Observatorio Turístico”, disponible el 2 de septiembre de 2017 en: http://www.dipucadiz.es/patronatoturismocadiz/observatorio-turistico.

Enríquez, J. G. y Casas, S. I. (2014): “Usabilidad en aplicaciones móviles”, Informes Científicos-Técnicos UNPA, vol. 5, nº 9, pp. 25-47.

Ferrá, F. C. M. y Cardona, J. R. (2015): "Presencia de las cadenas hoteleras españolas en los medios sociales”. CULTUR-Revista de Cultura y Turismo, vol. 9, n 1, pp. 05-35.

Foro Económico Mundial (2017): Travel \& Tourism Competitiveness Report, World Economic Forum, disponible en https://www.weforum.org/reports/the-traveltourism-competitiveness-report-2017.

Furroy, M. (2015): “¿Web responsive? Imprescindible para cualquier hotel en 2015”, Tecnohotel: revista profesional para la hostelería y restauración, $\mathrm{n}^{\circ} 465$, pp. 1617.

García, J. R. (2014): Organización, gestión y creación de empresas turísticas, Septem Ediciones, Oviedo.

González, Gil (2013): “Como hacer apps accesibles”, disponible el 15 de marzo de 2015 en:

http://www.ceapat.es/InterPresent1/groups/imserso/documents/binario/appsacces ibles.pdf.

González, D. y Marcos, M. C. (2013): "Responsive web design: diseño multidispositivo para mejorar la experiencia de usuario". BiD: textos universitaris de biblioteconomia i documentació, vol. 31, $\mathrm{n}^{\circ} 8$.

González, J. A. M. (2011): Marketing Turístico Online, Turismo y Desarrollo Local, vol. 4, n ${ }^{\circ}$ 9, pp. 1-13.

Hostalia (2012): “Cómo diseñar la landing page perfecta”, disponible el 2 de febrero de 2018 en: https://blog.hostalia.com/como-disenar-la-landing-page-perfecta.

Hostelsur (2017): "Hoteles: 18 claves para potenciar la venta directa y conseguir más ingresos”, disponible el 5 de febrero de 2018 en: https://www.hosteltur.com/123581_hoteles-18-claves-potenciar-venta-directaconseguir-ingresos.html.

IAB Spain (2017): “Estudio Anual del Mobile Marketing 2017”, disponible el 23 de septiembre de 2017 en: http://iabspain.es/wp-content/uploads/estudio-mobile2017-vcorta.pdf.

INTECO (Instituto Nacional de Tecnologías de la Comunicación): Centro de Referencia en Accesibilidad y Estándares Web (2009): “Accesibilidad en Contenidos Web para Móviles”, disponible el 28 de enero de 2018 en: http://sid.usal.es/centrosyservicios/discapacidad/14518/2-1-3/inteco-institutonacional-de-tecnologias-de-la-comunicacion.aspx. 
Leung, X. Y. y Tanford, S. (2016): "What drives facebook fans to "like" hotel pages: A comparison of three competing models", Journal of Hospitality Marketing \& Management, vol. 25, $\mathrm{n}^{\circ}$ 3, pp. 314-345.

López, J. M., López, L. M. y Sanz, B. (2009): "Las dimensiones de la búsqueda en la compra online del turista”, Administrando en entornos inciertos, vol. 16, pp. 4556.

Martín-Sánchez, M., Miguel-Dávila, J.A. y López-Berzosa, D. (2012): M-tourism: las apps en el sector turístico, IX Congreso Nacional Turismo y Tecnologías de la Información y las Comunicaciones (TURITEC), Málaga, España.

Mascaraque, E. S., Ocaña, A. M. y MARTOS, I. O. (2009): "Directrices técnicas referidas a la accesibilidad Web”, Anales de documentación, vol. 12, pp. 255280.

Miranda, D. D. P., Plaza, A. G. y Brea, J. A. F. (2016): “The investigation of business social responsibility in the hospitality sector. Analysis and review of the scientific literature”, Turismo y Sociedad, vol. 18, pp. 137-158.

Moreno, O. M., Contreras, T. C. y Sánchez, R. E. (2016): Examen de tendencias del turismo en el umbral del siglo XX, Competitive Press, México D.F.

Munar, A. M. (2012): "Social media strategies and destination management", Scandinavian Journal of Hospitality and Tourism, vol. 12, n 2, pp. 101-120.

Nielsen, J. y Budiu, R. (2013): Usabilidad en dispositivos móviles, Anaya Multimedia, Madrid.

Nielsen, J. y Loranger, H. (2006): Prioritizing web usability, Pearson Education, Washington.

Parra Luzuriaga, P. E. (2015): Diseño de interfaces Web Responsive para periódicos digitales (tesis de Máster), Universidad del Azuay.

Rekhi, S. (2013): "Square pegs and round holes: How to make e-learning more mobile responsive”, disponible el 5 de julio de 2107 en: http://saffroninteractive.com/square-pegs-and-round-holes.

Rodríguez, J. M., Alonso, M. M., Rubio, L., Esteban, C., Martín, J. I., Gil, S., y Figueroa, C. (2010): Estudio de las políticas de calidad aplicadas al sector turismo. Un análisis de las mejores prácticas en España y México, Visión Libros, Madrid.

Rodríguez, R. A., Marko, I., Vera, P. M., Valles, G. Y. y Acevedo Zain, G. (2017): Evaluación de la usabilidad en sitios web móviles adaptativos, XIX Workshop de Investigadores en Ciencias de la Computación, Buenos Aires.

Rojo, I. M. (2014): Dirección y gestión de empresas del sector turístico, Ediciones Pirámide, Madrid.

Sánchez, C. C. (2012): “Ciberperiodismo en el smartphone. Estudio de la multimedialidad, usabilidad, hipertextualidad e interactividad de las aplicaciones de medios nativos digitales para Smartphone”, Estudios sobre el mensaje periodístico, $\mathrm{n}^{\circ}$ 18, pp. 243-251.

Sharkie, C. y Fisher, A. (2013): Jump start responsive web design, Sitepoint Pty, Collingwood.

Shneiderman, B. (2010): Designing the user interface: strategies for effective humancomputer interaction, Pearson Education, Bangalore. 
Varela, F. y Shear, J. (2005): "Metodología en primera persona: qué, por qué, cómo”, Journal of consciousness Studies, vol. 6, n 2, 1-14.

W3C (2017): "Web accecibility initiative”, Disponible el 24 de mayo de 2017 en: http://www.w3.org/WAI/mobile.

Werthner, H., Alzua-Sorzabal, A., Cantoni, L., Dickinger, A., Gretzel, U., Jannach, D. y Stangl, B. (2015): "Future research issues in IT and tourism", Information Technology \& Tourism, vol. 15, n ${ }^{\circ}$ 1, pp. 1-15.

World Economic Forum (2017): “The Travel \& Tourism Competitiveness Report 2017”. Disponible el 14 de marzo de 2017 en: https://www.weforum.org/reports/the-travel-tourism-competitiveness-report2017

Zambrano, J. A. O., Cujilan, Y. T. C., Bernabe, M. D. C. T. y Castillo, K. N. L. (2017): "La usabilidad y accesibilidad: Estudio de guías para aplicaciones en dispositivos móviles”, Dominio de las Ciencias, vol. 3, nº 3, 1181-1209. 